\title{
O timização de sistema de autoaspiração de ar tipo Venturi para tratamento de água ferruginosa
}

\author{
Jeferson S. Piccin ${ }^{1}$, Alessander L. Rissini ${ }^{1}$, Jeferson J. Freddi' ${ }^{1}$, Marlo M. Koch ${ }^{1}$, Vandré B. Brião ${ }^{1} \&$ Marcelo Hemkemeier ${ }^{1}$
}

\section{RESU MO}

$\mathrm{Na}$ confecção deste trabalho se utilizou a metodologia de superfície de resposta para otimizar o efeito do número de Reynolds, tempo de floculação e concentração de hipoclorito de sódio sobre a oxidação/floculação do ferro presente em águas subterrâneas em um sistema de aeração com autoaspiração de ar. 0 sistema se compunha de um vaso tipo Venturi, acoplado a um tubo de mistura para promover a oxigenação da água através da sucção do ar atmosférico. 0 mapeamento hidrodinâmico permitiu verificar as condições de operação no qual o sistema apresentou melhor eficiência de sucção de ar e menor consumo de energia, além de compará-las com as melhores condições a campo. 0 s resultados observados demonstraram que foi possível a remoção de $98,7 \%$ do ferro presente (residual ferro de $0,06 \mathrm{mg} \mathrm{L}^{-1}$ ) quando

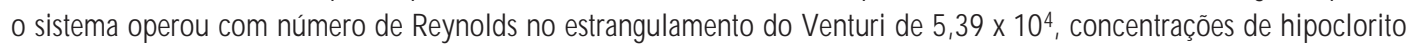
de sódio de 38,4 mg L-1 e tempo de floculação $30 \mathrm{~min}$. A metodologia de superfície de resposta foi satisfatória e permitiu otimizar as variáveis operacionais citadas.

Palavras-chave: aeração, águas subterrâneas, metodologia de superfície de resposta, remoção de ferro

\section{O ptimization of auto-aspiration aeration system type Venturi for the treatment of ferruginous water}

\begin{abstract}
In this study the response surface methodology was used to optimize the effect of Reynolds number, flocculation time and sodium hypochlorite concentration on the iron oxidation/flocculation present in groundwaters in an aeration system with air auto-aspiration. This system was composed of a recipient type Venturi coupled to a mixture tube to promote the oxygenation of the water through the suction of the atmospheric air. The hydrodynamic mapping allowed the verification of the operation conditions in which the system presented the best air suction efficiency and energy consumption, and the comparison of the best field conditions. The observed results demonstrated that it was possible to remove $98.7 \%$ of present iron (residual iron of $0.06 \mathrm{mg} \mathrm{L}^{-1}$ ) when the system operated with Reynolds number of $5.39 \times 10^{4}$, sodium hypochlorite concentrations of $38.4 \mathrm{mg} \mathrm{L}^{-1}$ and flocculation time of $30 \mathrm{~min}$. The response surface methodology was satisfactory and allowed for the optimization of the mentioned operational variables.
\end{abstract}

Key words: aeration, groundwater, surface response methodology, iron removal 


\section{INTRODUÇÃO}

O ferro é o quarto elemento mais abundante na crosta terrestre e pode ser facilmente encontrado em águas subterrâneas na forma de bicarbonato ferroso $\left[\mathrm{Fe}\left(\mathrm{HCO}_{3}\right)_{2}\right]$ (Sung \& Morgan, 1980). Sua presença em águas subterrâneas ocorre pela percolação da água em leitos de rochas que possuam materiais ferrosos em sua composição. O bicarbonato ferroso é solúvel, inodoro e incolor e, na presença de oxigênio e pH favorável, oxida-se, formando material precipitado, conferindo cor e sabor à água, podendo provocar sérios problemas na indústria e agricultura (Menezes et al., 2005; Michalakos et al., 1997; Souza et al., 2006).

Na indústria, a contaminação da água com minerais solúveis provoca, entre outros problemas, a diminuição da eficiência em trocadores de calor, rompimento de tubulações pela incrustação, aumento na rapidez da oxidação de superfícies metálicas expostas, alterações processuais e desenvolvimento de ferrobactérias (Menezes et al., 2005; Michalakos et al., 1997; Souza et al., 2006). Neste sentido se buscam, através de inúmeras pesquisas, alternativas para os sistemas de tratamento de águas contaminadas com ferro solúvel, como o desenvolvimento de resinas quelantes (Korngold, 1994), filtros de pedra (Michalakos et al., 1997), tratamento biológico (Katsoyiannis \& Zouboulis, 2004; Stembal et al., 2005), microfiltração (Ellis et al., 2000) e adsorção com resíduos de cinza (Das et al., 2007) e carvão (Ahamad \& Jawed, 2009), além dos sistemas tradicionais em que se promove a oxidação/floculação do ferro que é removido por filtração. Apesar de largamente utilizados, os sistemas tradicionais apresentam inúmeras desvantagens (Ellis et al., 2000) citando-se, como exemplos, a baixa eficiência de transferência de oxigênio, a necessidade de equipamentos e acessórios, operacionalidade complexa e grande área requerida para suas instalações.

Os sistemas de autoaspiração de ar são utilizados para promover a oxigenação da água; trata-se de um método que se baseia nos princípios do tubo de Venturi e se utiliza de um ejetor para misturar o oxigênio do ar com a água. Os ejetores são equipamentos capazes de transformar a energia de pressão do fluido primário em energia de velocidade que, por sua vez, poderá atingir um nível crítico que induz à formação de vácuo na seção de contração do Venturi, realizando a sucção do ar atmosférico através de um vaso paralelo ao escoamento (Teixeira, 1999; Baylar et al., 2007a.; Baylar et al., 2009). Assim, na aeração com ejetores tipo Venturi a oxigenação da água é produzida tanto por processo de ar dissolvido quanto por processo de ar disperso, sem limitações práticas da quantidade de ar que pode ser adicionada e sem a necessidade de compressores de ar, garantindo-se ótima eficiência com baixo investimento e podendo reduzir consumo energético (Gonçalves, 1999; Baylar et al., 2009).

Os trabalhos através de sistemas de autoaspiração de ar indicam que os fenômenos de transferência de massa nesses equipamentos ainda não são suficientemente conhecidos, apesar de quase seis décadas de pesquisa; isto se deve ao interesse de grande parte dos autores em estudar apenas o desempenho energético desses equipamentos, geralmente construídos de forma empírica e com várias particularidades. Este estudo deve ser executado avaliando-se, além dos parâmetros energéticos, os parâmetros adimensionais do sistema para assim se obter parâmetros de aumento de escala do sistema (Teixeira, 1999). Desta forma, Baylar \& Okzam (2006) reportam que a eficiência de transferência de oxigênio (E) demonstra a quantidade de oxigênio transferida pelo sistema em relação à quantidade possível de ser transferida; este termo é frequentemente utilizado para avaliar o desempenho de sistemas de aeração; já a razão entre as vazões volumétricas de água e o ar (Rz) serve como parâmetro de capacidade de sucção de oxigênio do equipamento (Teixeira, 1999).

Referente aos parâmetros energéticos, a taxa de transferência de oxigênio (TTO) e a eficiência de oxigenação (EO) se apresentam como as mais importantes, em virtude de reportarem a quantidade de oxigênio transferida por unidade de tempo e a quantidade de oxigênio transferida por quantidade de energia consumida (Sperling, 1997).

Neste trabalho se objetivou avaliar as características hidrodinâmicas de um Venturi tipo ejetor em relação à capacidade de sucção de ar e a eficiência de transferência de oxigênio e utilizar a metodologia de superfície de resposta para a otimização das variáveis operacionais deste sistema aplicado para o tratamento de águas subterrâneas contaminadas por ferro solúvel, buscando-se atingir concentrações de ferro total após o tratamento, que atendam aos níveis inferiores permitidos pela legislação vigente (Brasil, 2004).

\section{MATERIAL E MÉTODOS}

\section{Sistema utilizado}

O sistema foi composto de uma bomba centrífuga acionada por um motor de 1,1 kW (1,5 CV). A bomba foi acoplada a uma tubulação com diâmetro nominal de $32 \mathrm{~mm}$ (diâmetro útil de 25,4 mm), que conduzia o fluido até o sistema de autoaspiração de ar (Figura 1). À jusante e à montante do sistema de autoaspiração de ar foram acoplados dois manômetros de Bourbon para o controle da pressão de trabalho $\left(\mathrm{p}_{1}\right.$ e $\left.\mathrm{p}_{2}\right)$ e da queda de pressão do sistema. As medidas de vazão de água foram realizadas no fim da tubulação por cubagem. Perpendicularmente à linha do fluido primário, uma linha secundária promovia a condução de ar para o interior do ejetor; esta tubulação foi acoplada a um rotâmetro calibrado para ar, a fim de fornecer a leitura de vazão de ar e um manômetro para leitura da pressão de trabalho. Uma tubulação de 1 m de comprimento $\left(l_{\text {tubo }}\right)$ com misturadores estáticos acoplados em seu interior, conduzia e realizava a mistura da água e do oxigênio. Na parte superior do interior da tubulação de mistura foram instalados 5 misturadores estáticos, equidistantes entre si, confeccionado de acordo com Gonçalves (1999), com o objetivo de evitar a coalescência das bolhas de ar. A Figura 1 apresenta o esquema do sistema de aeração utilizado enquanto a Figura 2 mostra um desenho técnico do ejetor utilizado nos experimentos cujas medidas são apresentadas em milímetros; já a Tabela 1 apresenta as relações entre características geométricas do Venturi utilizado, que servem como parâmetro para aumento de escala. 


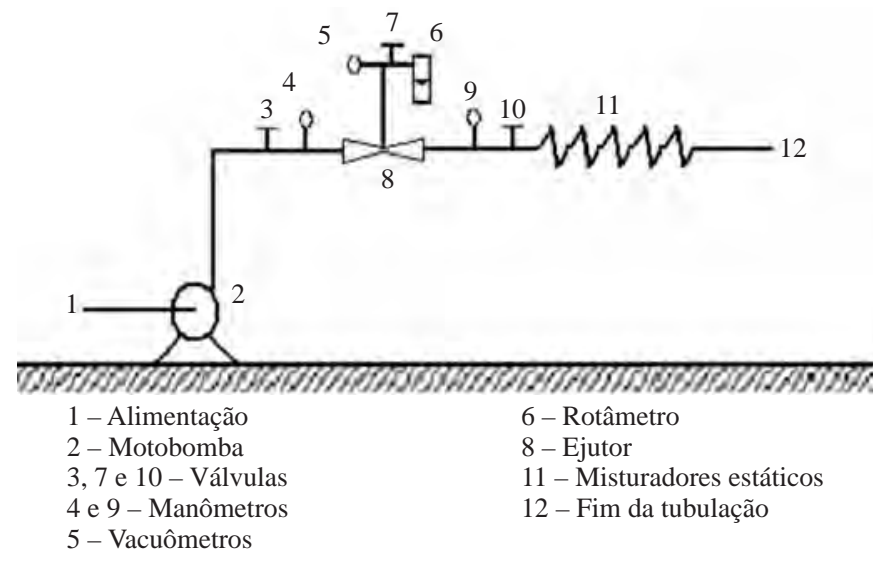

Figura 1. Esquema dos acessórios do sistema de aeração

Na Figura 2 e na Tabela 1, $\mathrm{d}_{\text {bico }}$ representa o diâmetro do vaso na região de estrangulamento máximo, $\mathrm{d}_{\mathrm{alim}}$. o diâmetro da tubulação de alimentação, $1_{\text {con. }}$ o comprimento da conicidade de alimentação, $\mathrm{d}_{\text {cam }}$ o diâmetro da câmara de sucção de ar, $l_{\mathrm{ar}}$ o espaço para a sucção do ar após o estrangulamento, $\mathrm{d}_{\mathrm{zm}}$ o diâmetro da zona de choque entre as bolhas de ar e a parede do tubo após o fim da conicidade,

Tabela 1. Características geométricas do ejetor

\begin{tabular}{|c|c|}
\hline Relação geométrica & Valor adotado \\
\hline $\mathrm{d}_{\mathrm{bico}} / \mathrm{d}_{\mathrm{alim}}$ & 0,16 \\
\hline $\mathrm{d}_{\mathrm{zm}} / \mathrm{I}_{\mathrm{con}}$ & 0,04 \\
\hline $\mathrm{d}_{\mathrm{bico}} / \mathrm{d}_{\mathrm{cam}}$ & 0,13 \\
\hline $\mathrm{d}_{\text {bico }} / \mathrm{d}_{\mathrm{ar}}$ & 0,67 \\
\hline $\mathrm{d}_{\mathrm{bico}} / \mathrm{l}_{\mathrm{ar}}$ & 8,00 \\
\hline $\mathrm{d}_{\mathrm{bico} 0} / \mathrm{d}_{\mathrm{zm}}$ & 0,40 \\
\hline $\mathrm{d}_{\mathrm{zm}} / \mathrm{l}_{\mathrm{zm}}$ & 0,05 \\
\hline $\mathrm{d}_{\text {bico }} / \mathrm{d}_{\text {tubo }}$ & 0,21 \\
\hline $\mathrm{d}_{\text {tubo }} / /_{\text {tubo }}$ & 0,02 \\
\hline$\alpha$ & $3^{\circ}$ \\
\hline
\end{tabular}

$\mathrm{l}_{\mathrm{zm}}$ o comprimento total da zona de choque, $\mathrm{d}_{\text {tubo }}$ o diâmetro do tubo de mistura, $l_{\text {tubo }}$ o comprimento do tubo de mistura e á o ângulo do Venturi, $\mathrm{h}_{\text {mist }}$ a altura do misturador estático e $l_{\text {mist }}$ o comprimento do misturador estático.

\section{Mapeamento hidrodinâmico}

Os testes de mapeamento hidrodinâmico foram realizados em laboratório, com auxílio de um reservatório em que a concentração de oxigênio dissolvido na água foi baixada para valores próximos a zero adicionando-se bissulfito de sódio (Ramalho, 1993). Os resultados observados da razão entre vazões volumétrica, eficiência de transferência de oxigênio, taxa de transferência de oxigênio e eficiência de oxigenação, foram relacionados ao número de Reynolds da água no estrangulamento do ejetor.

Para o cálculo da energia consumida pelo sistema (Eq. 1) considerou-se apenas a perda de carga do ejetor, sendo este o volume de controle adotado.

$$
\mathrm{E}=\frac{\left(\mathrm{p}_{2}-\mathrm{p}_{1}\right) \times \mathrm{m}_{\mathrm{H}_{2} \mathrm{O}}}{\rho_{\mathrm{H}_{2} \mathrm{O}}}
$$

em que $\rho_{\mathrm{H} 2 \mathrm{O}}$ é a densidade da água em $\mathrm{kg} \mathrm{m}^{-3}, \mathrm{p}_{1}$ e $\mathrm{p}_{2}$ são as pressões de trabalho na entrada e saída do ejetor em $\mathrm{kN} \mathrm{m}^{-2}$ e $\mathrm{m}_{\mathrm{H} 2 \mathrm{O}}$ é a vazão mássica de água em $\mathrm{kg} \mathrm{s}^{-1}$.

A análise de oxigênio dissolvido (OD) foi realizada pelo método de Winkler, descrito por APHA/AWWA/WPCF (2000), sendo que a diferença entre a concentração inicial e a final foi utilizada para o cálculo da quantidade do oxigênio transferido.

\section{Aplicação na remoção de ferro em águas subterrâneas \\ Realizou-se a aplicação do sistema em estudo na remo- ção do ferro solúvel em águas subterrâneas, em um poço artesiano da região de Passo Fundo, RS, que possui níveis de ferro solúvel acima dos padrões exigidos pela Portaria 518/04 do Ministério da Saúde. Alíquotas de água oxigenada}

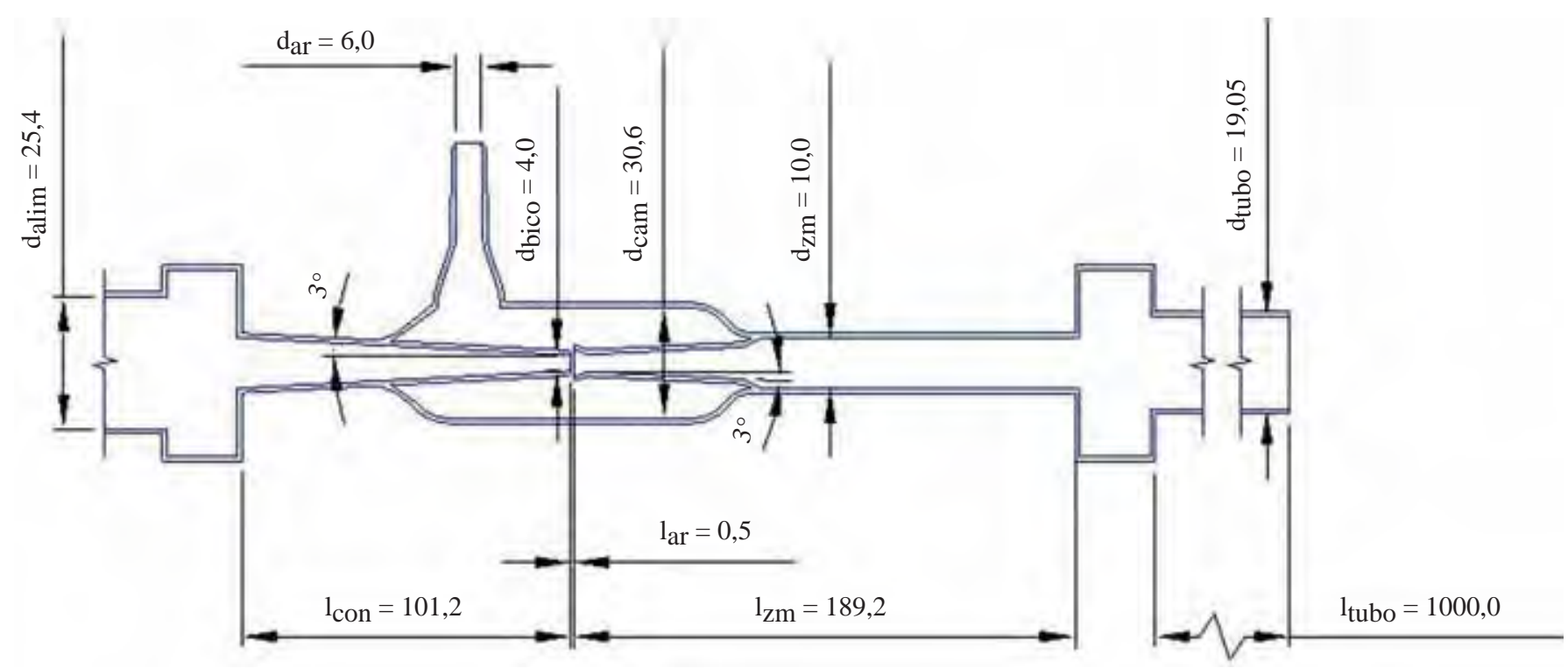

Figura 2. Desenho técnico do ejetor utilizado 
pelo ejetor foram coletadas em erlemeyers no fim da tubulação de mistura, adicionando-se a solução de hipoclorito de sódio, que foi deixada em repouso para ocorrer a oxidação do ferro e a floculação; amostras eram retiradas dessas alíquotas em tempos preestabelecidos, filtradas em papel filtro Watman $n^{\circ} 40$ e armazenadas em tubos de ensaios, hermeticamente fechados para posterior análise do ferro total. Realizaram-se as determinações de ferro total de acordo com o método da ortofenantrolina (APHA/AWWA/WPCF, 2000) em um fotômetro, marca Merck, modelo SQ 118.

Os experimentos foram realizados de acordo com um delineamento composto central rotacional (DCCR), conforme descrito por Box et al. (1978), em que as variáveis operacionais estudadas foram o número de Reynolds no estrangulamento do Venturi $\left(\mathrm{N}_{\mathrm{Re}}\right)$, tempo de oxidação/floculação $(\mathrm{t})$ e concentração de hipoclorito de sódio ([NaClO]). Os níveis das variáveis utilizadas estão descritos na Tabela 2.

Tabela 2. M atriz dos valores codificados e níveis das variáveis utilizadas no DCCR para a remoção de ferro

\begin{tabular}{cccc}
\hline Nível & $\mathbf{N}_{\mathrm{Re}} \times \mathbf{1 0}$ & $\mathbf{t}(\mathbf{m i n})$ & $\begin{array}{c}{[\mathbf{N a C l 0}]} \\
\left(\mathbf{m g ~ L} \mathbf{L}^{-1}\right)\end{array}$ \\
$-1,68$ & 4,33 & 14,2 & 0,00 \\
-1 & 5,39 & 20,0 & 7,68 \\
0 & 6,72 & 30,0 & 19,20 \\
+1 & 8,05 & 40,0 & 30,72 \\
$+1,68$ & 8,89 & 46,8 & 38,40 \\
\hline
\end{tabular}

Os experimentos foram relacionados com o percentual de ferro removido, obtido pela relação entre a diferença da concentração inicial e final e a concentração inicial, enquanto se realizaram análises estatísticas com auxílio de software computacional livre. Todas as soluções utilizadas possuíam padrão analítico.

\section{RESULTADOS E DISCUSSÃO}

A Figura 3 apresenta os resultados obtidos no mapeamento hidrodinâmico do sistema de autoaspiração de ar em relação ao número de Reynolds no estrangulamento do Venturi.

As razões entre as vazões volumétricas de ar e água (Rz) variavam de 0,15 a 0,53 quando o sistema operou com número de Reynolds no estrangulamento $\left(\mathrm{N}_{\mathrm{Re}}\right)$ de $3,9 \times 10^{4}$ e $8,1 \times 10^{4}$, respectivamente, nas quais de deu a maior amplitude, fato evidenciado nos experimentos. Através da análise dos valores da Rz vs Re, Figura 3A, foi possível observar que valores de Re inferiores a até 8,0 x $10^{4}$ a Rz aumentou e, após este valor, voltou a diminuir; tal comportamento se deve ao aumento da perda de carga na tubulação de sucção, ejetor e tubo de mistura, que reduz os valores da razão de sucção de ar (Gonçalves, 1999). Segundo Teixeira (1999) os valores ótimos para esta relação devem variar entre 0,6 a 0,8 , os quais não foram obtidos com o sistema utilizado. Os resultados obtidos por Baylar \& Ozkan (2006) e Ozkan et al. (2006) demonstraram que a relação entre as vazões de ar e água diminuiu com o aumento da velocidade no estrangula-

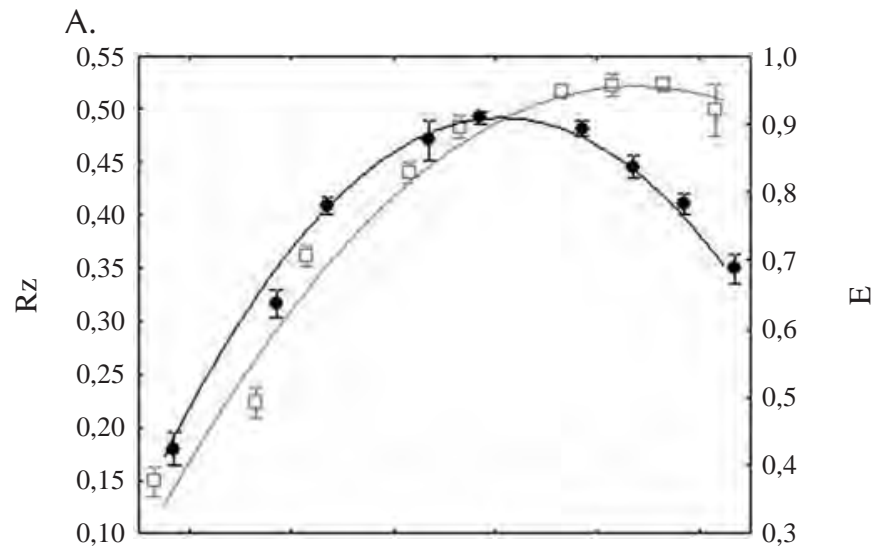

B.

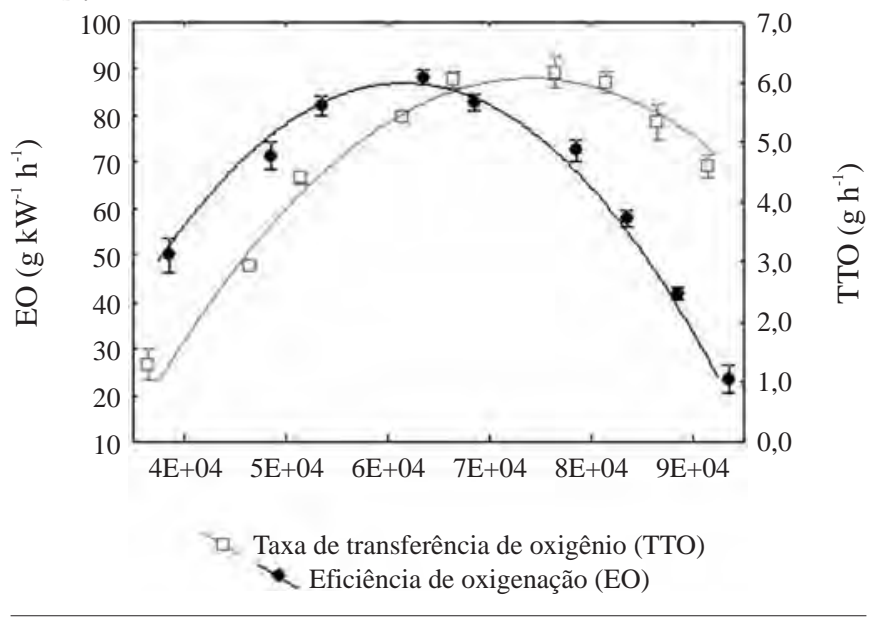

Figura 3. Mapeamento hidrodinâmico em relação ao número de Reynolds no estrangulamento: (A) razão entre vazão de ar e água (Rz) e eficiência de transferência de oxigênio (E); (B) Eficiência de oxigenação (EO) e taxa de transferência de oxigênio (TTO)

mento, por consequência do número de Reynolds, e o aumento do diâmetro de entrada do Venturi. Baylar et al. (2007b.) observaram este mesmo comportamento porém com valores de razão entre vazões inferiores a 0,35. Nesses mesmos resultados foi possível observar que a redução da relação entre o diâmetro do tubo e o diâmetro do bico de 0,5 para 0,75 provocou redução significativa na razão entre as vazões; já Gourich et al. (2007) notaram que a razão entre vazão máxima foi de 0,4 quando o sistema operava com número de Reynolds no estrangulamento de 4,0 x $10^{4}$.

Os resultados obtidos para a eficiência de transferência de oxigênio (E) variaram entre 0,40 e 0,90, Figura 3A, quando o sistema operou com o número de Reynolds $\left(\mathrm{N}_{\mathrm{Re}}\right)$ entre $3,9 \times 10^{4}$ e $7,5 \times 10^{4}$, respectivamente; referidos resultados reportam que o equipamento foi capaz de promover a aeração da água a valores próximos a saturação. Da mesma forma que a razão entre vazões (Rz), com valores de Re inferiores a 6,5 x $10^{4}$ a eficiência de transferência de oxigênio (E) aumentou com o aumento do Re, permanecendo constante entre $6,5 \times 10^{4}$ e $8,0 \times 10^{4}$ e diminuindo após esses valores, Figura 3A. Baylar \& Ozkan (2006) obtiveram valores para a eficiência de transferência de oxigênio (E) em torno de 0,57 , valores muito inferiores aos obtidos nesta pesquisa. $\mathrm{O}$ aumento da eficiência de transferência de oxigênio pode ter 
sido influenciado pelas características geométricas e operacionais do sistema, pelo comprimento e diâmetro ou pelas dimensões do tubo de mistura e, ainda, pela utilização de misturadores estáticos.

Em relação à eficiência de oxigenação (EO), o melhor resultado foi de $90,1 \mathrm{~g} \mathrm{~kW}^{-1} \mathrm{~h}^{-1}$ com número de Reynolds $\left(\mathrm{N}_{\mathrm{Re}}\right)$ 6,1 x 104. Da mesma forma, os valores de EO aumentaram até quando o número de Reynolds no estrangulamento foi inferior a $6,3 \times 10^{4}$ sendo que, a partir deste ponto, a tendência de redução da EO se deve à maior perda de carga e à menor eficiência na transferência de oxigênio (E). Os resultados obtidos por Gonçalves (1999) de 140,0 $\mathrm{g} \mathrm{kW}^{-1} \mathrm{~h}^{-1}$, não levaram em consideração o consumo de energia desperdiçado para vencer o obstáculo oferecido ao fluido pelo Venturi, inerente ao processo.

Os resultados obtidos para a taxa de transferência de oxigênio (TTO) em relação ao número de Reynolds no estrangulamento $\left(\mathrm{N}_{\mathrm{Re}}\right)$ variaram entre 1,1 a $6,4 \mathrm{~g} \mathrm{~h}^{-1}$ quando o sistema operava com número de Reynolds no estrangulamento de $3,9 \times 10^{4}$ e $7,4 \times 10^{4}$, respectivamente. A partir da análise dos valores da TTO vs Re (Figura 3B), foi possível observar que a TTO aumenta para valores de Re inferiores a $7,5 \times 10^{4}$, diminuindo a partir desse valor, comportamento semelhante ao apresentado pela razão entre vazões de ar e água (Rz), conforme demonstrado na Figura 3A, evidenciando a citação de Teixeira (1999).

A caracterização da água do poço artesiano utilizado para a aplicação do sistema a campo, mostrou que a concentração de ferro foi de $4,1 \pm 0,1 \mathrm{mg} \mathrm{L}^{-1}$, a qual se apresentava completamente incolor na saída do poço, porém era oxidada quando em contato com o ar atmosférico por pequeno período de tempo, formando um material de cor alaranjada, conforme descrito por Michalakos et al. (1997). Este comportamento, aliado ao $\mathrm{pH}$ abaixo de 7,0, indica a presença de ferro na forma de bicarbonato ferroso $\left(\mathrm{Fe}\left(\mathrm{HCO}_{3}\right)_{2}\right)$.

A Tabela 3 apresenta o delineamento experimental utilizado e o percentual de remoção nos experimentos de otimização das variáveis operacionais número de Reynolds no estrangulamento do Venturi $\left(\mathrm{N}_{\mathrm{Re}}\right)$, tempo de oxidação/floculação (t) e concentração de hipoclorito de sódio ([NaClO]).

A Tabela 4 apresenta os resultados obtidos a partir da análise estatística realizada sobre os resultados apresentados na Tabela 3.

A Tabela 4 mostra que todos os efeitos, tanto lineares como quadráticos, se apresentaram significativos para um grau de significância de 95\% (p<0,05). As interações entre as variáveis não se mostraram significativas; desta forma, a retirada dos parâmetros não significativos provoca o aumento dos graus de liberdade da falta de ajuste, reduzindo sua significância. A Tabela 5 apresenta a análise de variância do modelo estatístico, realizada com a retirada dos fatores não significativos para verificar se o modelo explica as variações observadas nas respostas.

Os resultados observados demonstram que o $\mathrm{F}_{\text {calc, }}$ para uma distribuição de Fischer com 6 graus de liberdade para a regressão e 10 graus de liberdade para os resíduos, a um nível de significância de 95\%, foi 5,7 vezes maior que o valor tabelado, indicando que o modelo é preditivo e capaz de
Tabela 3. Matriz do DCCR com valores reais das variáveis e as respostas de percentual de ferro removido

\begin{tabular}{|c|c|c|c|c|}
\hline Exp. & $\mathrm{N}_{\mathrm{Re}} \times 10^{-4}$ & $t(\min )$ & $\begin{array}{l}{[\mathrm{NaClO}]} \\
\left(\mathrm{mg} \mathrm{L}^{-1}\right)\end{array}$ & $\begin{array}{l}\text { Remoção } \\
\text { de ferro (\%) }\end{array}$ \\
\hline 1 & 5,39 & 20,0 & 7,68 & 86,2 \\
\hline 2 & 8,05 & 20,0 & 7,68 & 86,1 \\
\hline 3 & 5,39 & 40,0 & 7,68 & 87,5 \\
\hline 4 & 8,05 & 40,0 & 7,68 & 89,7 \\
\hline 5 & 5,39 & 20,0 & 30,72 & 91,2 \\
\hline 6 & 8,05 & 20,0 & 30,72 & 91,2 \\
\hline 7 & 5,39 & 40,0 & 30,72 & 93,0 \\
\hline 8 & 8,05 & 40,0 & 30,72 & 93,2 \\
\hline 9 & 4,33 & 30,0 & 19,20 & 84,0 \\
\hline 10 & 8,98 & 30,0 & 19,20 & 90,2 \\
\hline 11 & 6,72 & 14,2 & 19,20 & 92,8 \\
\hline 12 & 6,72 & 46,8 & 7,68 & 91,0 \\
\hline 13 & 6,72 & 30,0 & 0,00 & 87,7 \\
\hline 14 & 6,72 & 30,0 & 38,40 & 98,7 \\
\hline 15 & 6,72 & 30,0 & 19,20 & 94,5 \\
\hline 16 & 6,72 & 30,0 & 19,20 & 94,3 \\
\hline 17 & 6,72 & 30,0 & 19,20 & 94,7 \\
\hline
\end{tabular}

Tabela 4. Análise estatística dos resultados de otimização do consumo de hipoclorito

\begin{tabular}{lrrrrc}
\hline Efeito & \multicolumn{1}{c}{ S.Q. } & G.L. & Q.M. & \multicolumn{1}{c}{$\mathbf{F}$} & p \\
$\mathrm{N}_{\mathrm{Re}}(\mathrm{L})$ & 9,17 & 1 & 9,168 & 191,47 & 0,0052 \\
$\mathrm{~N}_{\mathrm{Re}}(\mathrm{Q})$ & 89,20 & 1 & 89,201 & 1862,94 & 0,0005 \\
$\mathrm{t}(\mathrm{L})$ & 3,18 & 1 & 3,183 & 66,48 & 0,0147 \\
$\mathrm{t}(\mathrm{Q})$ & 16,40 & 1 & 16,396 & 342,44 & 0,0029 \\
{$[\mathrm{NaClO}$ (L) } & 103,09 & 1 & 103,085 & 2152,92 & 0,0005 \\
{$[\mathrm{NaClO}](\mathrm{Q})$} & 5,25 & 1 & 5,253 & 109,71 & 0,0090 \\
$\mathrm{~N}_{\mathrm{Re}} \times \mathrm{t}$ & 0,79 & 1 & 0,792 & 16,53 & 0,0555 \\
$\mathrm{~N}_{\mathrm{Re}} \times[\mathrm{NaClO}]$ & 0,43 & 1 & 0,432 & 9,03 & 0,0952 \\
$\mathrm{t} \times$ [NaClO] & 0,18 & 1 & 0,181 & 3,78 & 0,1913 \\
Falta de ajuste & 17,66 & 5 & 3,532 & 73,76 & 0,0134 \\
Erro puro & 0,10 & 2 & 0,048 & & \\
Total & 229,23 & 16 & & & \\
\hline
\end{tabular}

Tabela 5. Análise de variância para o modelo estatístico gerado

\begin{tabular}{crrrcc}
\hline Parâmetro & S.Q. & G.L. & Q.M. & Fcalc. & Ftab. \\
Regressão & 210 & 6 & 35,0 & 18,3 & 3,2 \\
Resíduos & 19 & 10 & 1,9 & & \\
\hline
\end{tabular}

explicar as variações observadas nos experimentos (Box et al., 1978). O valor do coeficiente de correlação $\left(\mathrm{R}^{2}\right)$ foi superior a 0,9, sinal de que o modelo é capaz de explicar as variações dos dados experimentais. A Eq. 6 apresenta o modelo estatístico gerado.

Remoção de ferro(\%) $=4,12+2,0610^{-3} \times \mathrm{N}_{\mathrm{Re}}-1,4810^{-8} \times \mathrm{N}_{\mathrm{Re}}{ }^{2}+$ $0,81 \times \mathrm{t}-0,01 \times \mathrm{t}^{2}+0,44 \times[\mathrm{NaClO}]-5,2210^{-3} \times[\mathrm{NaClO}]^{2}$

A partir do modelo apresentado na Eq. 2, geraram-se as superfícies de respostas da remoção de ferro em relação às variáveis estudadas apresentadas na Figura 4, na qual foi mantido, como variável fixa, o tempo de floculação de 20 min, Figura 4A, a fim de apresentar a viabilidade de 


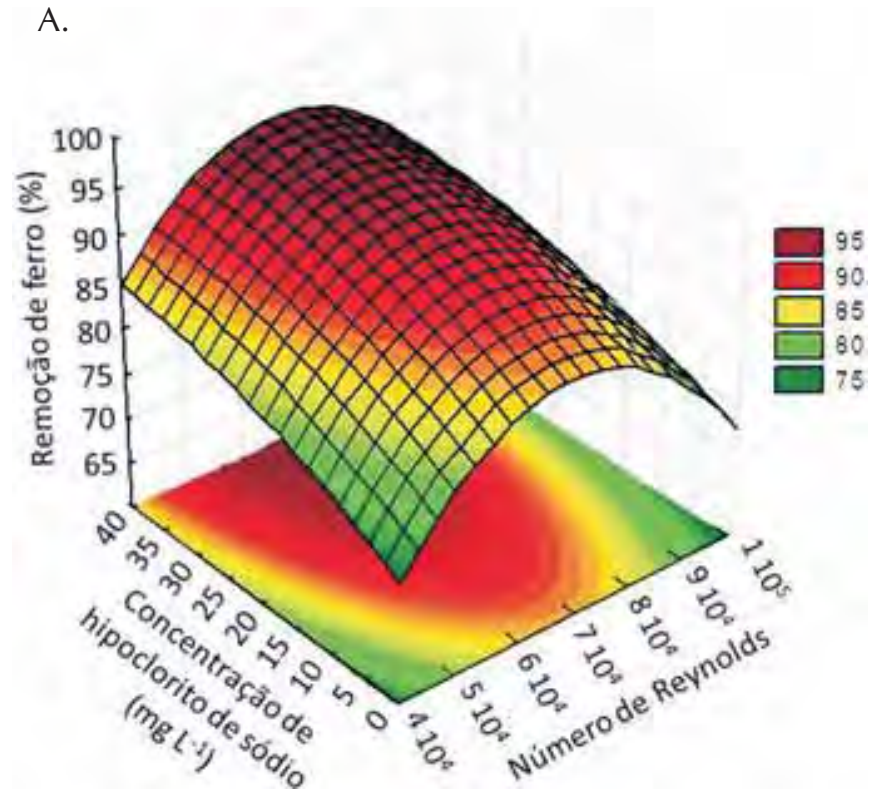

B.

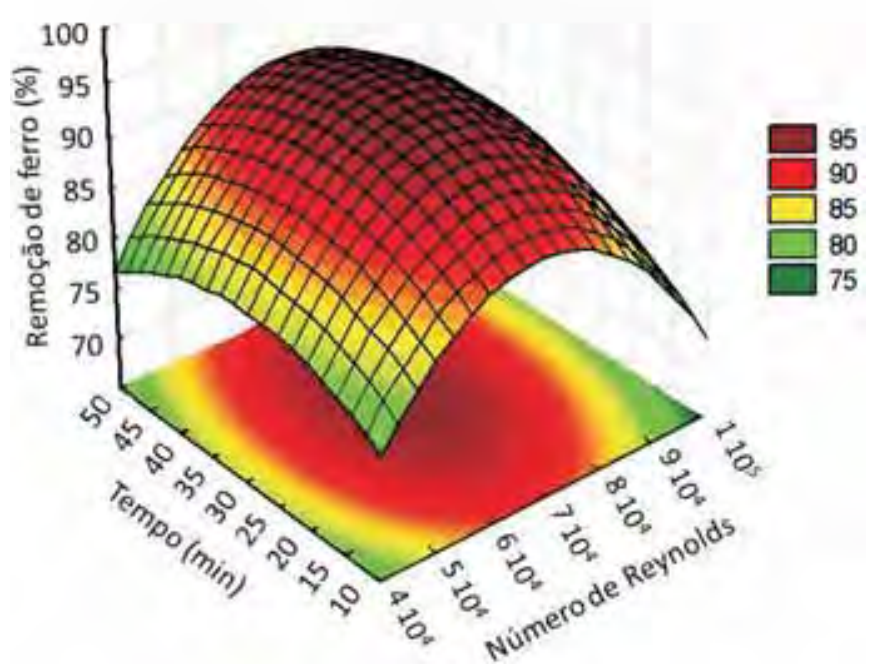

Figura 4. Superfície de resposta gerada a partir do modelo estatístico para a remoção de ferro: Tempo de floculação de 20 min (A) e concentração de hipoclorito de sódio de 19,2 $\mathrm{mg} \mathrm{L}^{-1}$ (B)

redução do tempo de floculação e concentração de hipoclorito de sódio de 19,2 $\mathrm{mg} \mathrm{L}^{-1}$, Figura 4B, para demonstrar a possibilidade de redução de consumo de hipoclorito de sódio.

Na Figura 4A é possível observar que a utilização de tempo de floculação de $20 \mathrm{~min}$, concentrações de hipoclorito de sódio de $30 \mathrm{mg} \mathrm{L}^{-1}$ e Reynolds no estrangulamento de $7,0 \times 10^{4}$, são suficientes para a remoção através de sistemas de filtros adequados de $95 \%$ do ferro existente na água. Observa-se, na Figura 4B que, com concentrações de hipoclorito de sódio de 19,2 mg L-1, número de Reynolds em torno de $6,5 \times 10^{4}$ a $7,5 \times 10^{4}$ e tempos de floculação superiores a 25 min, se obtêm remoções de ferro superiores a 94\% através de filtros adequados; quando comparados com os resul- tados obtidos por Korngold (1994), nota-se que os resultados foram superiores nas melhores condições de operação do sistema, sendo que os tempos de oxidação e floculação foram inferiores ao tempo de tratamento utilizado com o uso de resina de troca iônica.

O aumento na concentração de hipoclorito de sódio promoveu acréscimo na área em que a oxidação do ferro é satisfatória. Sung \& Morgan (1980) e Cho (2005) descrevem que o ânion hipoclorito se hidrolisa, formando ácido hipocloroso, que possui elevado poder oxidante e o íon $\mathrm{Na}^{+}$atua como base de Lewis, aumentando o pH da solução e facilitando a oxidação do ferro (Sung \& Morgan, 1980; Cho, 2005; Souza et al., 2006).

Quando comparados, os resultados do número de Reynolds do tratamento ao mapeamento hidrodinâmico do sistema, comprovam as expectativas de que a utilização de sistemas de aeração eficiente diminui a necessidade de adição de hipoclorito para que ocorra o processo de oxidação e floculação, pois os melhores resultados observados foram obtidos com número de Reynolds de $6,5 \times 10^{4}$ a 8,0 x $10^{4}$. Constatou-se, nesta faixa de valores o ponto ótimo do sistema tendo em vista a redução do consumo de hipoclorito de sódio e, por consequência, dos custos, onde se obtiveram valores teóricos da remoção iguais a $100 \%$ com operação a um número de Reynolds de $6,9 \times 10^{4}$, tempos de floculação de 40,5 min e concentração de hipoclorito de $25,3 \mathrm{mg} \mathrm{L}^{-1}$, condições em que é possível se obter vazões de trabalho neste equipamento, de $725 \mathrm{~L} \mathrm{~h}^{-1}$.

\section{CONCLUSÕES}

1. O mapeamento hidrodinâmico do sistema permitiu obter parâmetros para dimensionamento do consumo energético do sistema de autoaspiração de ar, e contribuiu na otimização do processo a campo.

2. O sistema foi capaz de reduzir a concentração de ferro solúvel a valores inferiores a $0,3 \mathrm{mg} \mathrm{L}^{-1}$, atendendo aos padrões da Portaria 518/04 do Ministério da Saúde.

3. A metodologia de planejamento experimental foi satisfatória para a otimização das variáveis operacionais do sistema.

4. Os melhores resultados observados foram obtidos com número de Reynolds $\left(\mathrm{N}_{\mathrm{Re}}\right)$ de 6,5 x $10^{4}$ a 8,0 x $10^{4}$, tanto na questão hidrodinâmica do sistema quanto como na oxidação do ferro solúvel.

\section{LITERATURA CITADA}

Ahamad, K.U.; Jawed, M. Kinetics, equilibrium and breakthrough studies for Fe(II) removal by wooden charcoal: A low-cost adsorbent. Desalination, v.251, p.137-145, 2009.

APHA/AWWA/WPCF- American Public Health Association; American Water Work Association; Water Pollution Control Facilities. Standard methods for the examination of water and wastewater. Washington: American Public Health Association, 20th, 2000, CD-Rom 
Baylar, A.; Ozkan, F. Applications of Venturi principles to water aeration systems. Environmental Fluid Mechanics, v.6, p.341-357, 2006.

Baylar, A.; Unsal, M.; Ozkan, F. On the use of Venturi tubes in aeration. Clean, v.35, n.2, p.183-185, 2007a.

Baylar, A.; Unsal, M.; Ozkan, F. Determination of the optimal location of the air hole in Venturi aerators. Clean, v.35, n.3, p.246-249, 2007b.

Baylar, A.; Unsal, M.; Ozkan, F. Hydraulic structures in water aeration processes. Water, Air and Soil Pollution, v.203, p.1-14, 2009.

Box, E. G. P.; Hunter, W. G.; Hunter, J. S. Statistics for experiments: An introduction to designs, data analysis and model building. New York: Wiley, 1978. 653p.

Brasil. Portaria n. 518, de 25 de março de 2004. Padrão de potabilidade da água para consumo humano. Diário Oficial da República Federativa do Brasil, Brasília, DF. 19p.

Cho, B. Y. Iron removal using an aerated granular filter. Process Biochemistry, v.40, p.3314-3320, 2005.

Das, B.; Hazarika, P.; Saikiaa, G.; Kalitab, H.; Goswamib, D. C.; Dasb, H. B.; Dubeb, S. N.; Duttaa, R. K. Removal of iron from groundwater by ash: A systematic study of a traditional method. Journal of Hazardous Materials, v.141, n.3, p.834-831, 2007.

Ellis, D.; Bouchard, C.; Lantagne, G. Removal of iron and manganese from groundwater by oxidation and microfiltration. Desalination, v.130, p.255-264, 2000.

Gonçalves, M. A. B. Estudo de transferência de oxigênio em reatores Air-Lift utilizando Venturi e peças cisalhantes. Campinas: UNICAMP, 1999. 144p. Tese Doutorado

Gourich, B.; Azher, N.; Vial, C.; Soulami, M. B.; Ziyad, M.; Zoulalian, A. Influence of operating conditions and design parameters on hydrodynamics and mass transfer in an emulsion. Chemical Engineering and Processing, v.46, p139-149, 2007.

Katsoyiannis, I. A.; Zouboulis, A. I. Biological treatment of Mn (II) and $\mathrm{Fe}$ (II) containing groundwater: kinetic considerations and product characterization. Water Research, v.38, p.1922-1932, 2004.
Korngold, E. Iron removal from tap water by a cation exchanger. Desalination, v.94, p.243-249, 1994.

Menezes, A. C. L. S. M.; Gadelha, C. L. M.; Silva Júnior, W. R.; Machado, T. T. V.; Almeida, T. M. V. Caracterização de água de lavagem de uma estação de tratamento de água, com vistas ao reuso. Revista Brasileira de Engenharia Agrícola e Ambiental, v.9, p.191-196, 2005.

Michalakos, G. D.; Nieva, J. M.; Vayenas, D. V.; Lyberatos, G. Removal of iron from potable water using a trickling filter.Water Research, v.31, n.5, p.991-996, 1997.

Ozkan, F.; Ozturk, M.; Baylar, A. Experimental investigations of air and liquid injection by Venturi tubes. Water and Environment Journal, v.20, p.114-122, 2006.

Ramalho, R. S. Tratamiento de águas residuales. Barcelona: Reverté S. A., 1993. cap.4, p.199-252.

Souza, J. A. A.; Cordeiro, E. A.; Costa, E. L. Aplicação de hipoclorito de sódio para a recuperação de gotejadores entupidos em irrigação com água ferruginosa. Revista Brasileira de Engenharia Agrícola e Ambiental, v.10, n.1, p.5-9, 2006.

Sperling, M. von. Princípio do tratamento biológico de águas residuárias: Lodos ativados. Belo Horizonte: UFMG.4, 1997. cap.5, p.127-161.

Stembal, T.; Markic, M.; Ribicic, N.; Briski, F.; Sipos, L. Removal of ammonia, iron and manganese from groundwaters of northern Croatia-pilot plant studies. Process Biochemistry, v.40, n.1, p.327-335, 2005.

Sung, W.; Morgan, J. J. Kinetics and product of ferrous iron oxygenation in aqueous systems. American Chemical Society: v.14, n.5, p.561-568, 1980.

Teixeira, M. B. F. Hidrodinâmica e transferência de massa em ejetores líquido-gás com escoamento descendente. Campinas: UNICAMP, 1999. 146p.Dissertação Mestrado 